\title{
Interface and Visualization Metaphors
}

\author{
Vladimir Averbukh1, 1, Mikhail Bakhterev ${ }^{1}$, Aleksandr Baydalin1, \\ Damir Ismagilov, 2 and Polina Trushenkova 2 \\ 1 Inst. Math. Mech. 16. S. Kovalevskoi, Yekaterinburg. Russia. 620219 \\ averbukh@imm.uran.ru, mike.bakhterev@gmail.com, bajur@imm.uran.ru \\ 2 Ural State University, 51. Lenina, Yekaterinburg, Russia. 620083 \\ idruz@mail.ru, trushenkova.p@gmail.com
}

\begin{abstract}
The paper is devoted to problems of computer metaphors,, such as Interface metaphor and Visualization metaphor. Interface metaphor is considered as the basic idea of likening between interactive objects and model objects of the application domain. A visualization metaphor is defined as a map establishing the correspondence between concepts and objects of the application domain under modeling and a system of some similarities and analogies. This map generates a set of views and a set of methods for communication with visual objects. Some positions of the metaphor theory are discussed. Concept of metaphor action is suggested.. "Formula" of metaphor is constructed. A set of examples of metaphor was analyzed. Aprioristic quality criteria of interface and visualization metaphors are suggested. These criteria allow evaluating as existing metaphors and to search for adequate metaphors for designing new specialized systems.
\end{abstract}

Keywords: Computer Metaphor, Visualization, Interface

\section{Introduction}

This paper continues our researches of visualization metaphors that results have been published in [1-5], In turn these researches have been initiated by problems arising during processes of design and development of specialized visualization systems. Specificity of such systems frequently demands new methods of visualization and interaction that are adequate to the given task and concrete (possibly narrow) user's classes. The practice of design and development of specialized visualization systems shows necessity of specific metaphors, and a stage of metaphor searching and/or designing is a part of development process. Our goal is to design well the visual interactive systems, and choice of good (adequate) metaphors is the key to proper decisions.

Below some points of the metaphor theory are considered in connection with computer metaphors. The structural analysis of concrete metaphors is carried out. This analysis is necessary to understand the reasons of successes of one and failures of other visualization and visual interface metaphors. Studying of visualization object structures together with understanding of logic of metaphor choice and/or generation 
may help to formulate criteria of evaluations of cognitive component of visual systems.

\section{Related works}

The most part of literature on this theme reflects researches and development on interface metaphors. The important generalizing results contain in [8] where a today state of the interface metaphor theory and practice is described in detail. Metaphor is considered as use everyday and/or well known (often technical) realities. Thus the concept of visual interface metaphor is based on presentation new or unusual for an user phenomena by means of other phenomena, that are known to him of everyday life. These phenomena should have the same basic properties, as the phenomena, which they explain [15]. Thereby constraints of metaphor habitualness and completeness are brought forth [16].

In [7] problems of metaphor interpretation are given much attention. Interpretation problems are considered both for the designer of an interactive system and for its) user.

In [17] the ideas metaphor formalization are of an interest. Note, that the first works on formalization in this area have appeared in the beginning of 90th years. (See, for example, [12].)

Statement (and a partial decision) of problems of metaphor search automatic and semi-automatic metaphor generation are considered in [10] and [6].

As a rule, in the literature the concept of a visualization metaphor is not separated from the concept of human-computer interface metaphor. In turn our attention has been directed on visualization metaphors. Earlier we have been emphasized the difference between interface and visualization metaphors [1]. Now it is important to show not only the difference, but also the conceptual unity of these types of computer metaphors. The semiotic analysis of visual interface and examples of visualization may reveal in both cases the existence of sign systems as a basis of interactions and communications. On our opinion these sign systems are generated by computer metaphors. Some theoretical aspects of metaphor are considered in the next issue.

\section{Computer Metaphors}

The last decades metaphors are considered not only from traditional positions of philology, but also philosophies, logic, cognitive sciences, psychologies, and computer sciences. A basis of our consideration of metaphor conception is approaches to studying a scientific metaphor advanced by T. Kuhn, as well as the cognitive approach to metaphors, advanced by J. Lakoff. [11,13-14]

A role of a scientific metaphor in development of scientific disciplines is revealed and described (see, [11] and the detailed book [9]). Also there is well known by J. Lakoff and M. Johnson book [14] devoted to a role of metaphors in a modern society and its universal penetration into science, politics, daily activity and thinking of people. 
The metaphor in this connection is considered as use of concepts and knowledge from one (source) area of human experience to understand better and to structure the phenomena and concepts another (target) domain that as a rule is more abstract.

Computer sciences, as well as all new scientific disciplines developed with active using of metaphors. The most known, for example, such as "memory" or "file" almost was lost links with initial concepts. The problems of computer metaphor become the topic of a large body of research. Further we consider two types of a computer metaphor - interface metaphor and visualization metaphor.

Interface metaphor is considered as the basic idea of likening between interactive objects and model objects of the application domain. Its role is to promote the best understanding of semantics of interaction, and also to determine the visual representation of dialog objects and a set of user manipulations with them.

Specificity of visualization, as independent discipline in frameworks of Computer Sciences, demands the distinction between visualization metaphors and interface metaphors. The concept of visualization metaphor is defined for generalization of metaphor using cases in all domains of Computer Visualization.

Let's define a visualization metaphor as a map establishing the correspondence between concepts and objects of the application domain under modeling and a system of some similarities and analogies. This map generates a set of views and a set of methods for communication with visual objects. We consider the metaphoricalness of any visualization.

This approach is substantially based on the concepts of semiotics. In terms of semiotics the metaphor is something dynamic, in contrast to a stable sign. We can describe a metaphor as the act or the process of a designation of one concept by means of a sign, traditionally connected to other concept. The visual interface uses regularly the language based on one or other sign system. Human-computer interaction in this connection may be described precisely as sign process. The analysis of examples shows, that visualization also may be described as sign process similarly to human-computer interaction. The choice of a metaphor is a choice of a sign system. This sign system is used to define the dialog language of interaction and/or visualization. Another function of a metaphor is to determine the context for a correct interpretation of language elements, and to reveal the sense of visual texts. Thus, interface and visualization metaphors provide understanding represented entities of the application domain, and also metaphors help to create new entities based on the internal metaphor logic.

Understanding of metaphors as mapping from source to target domains is incomplete in case of a visualization metaphor.

We offer more complex mechanism, which underlies functioning of metaphors. Our approach differs from others that in its frameworks the metaphor generates some independent metaphor domain at the expense of correspondence that puts to objects of target domain some objects from the source domain. And more exactly, structures and/or characteristics of objects from target domain are put in the correspondence structures and characteristics of objects from source domain. Cite an example of a classical metaphor LIFE IS A JOURNEY, where LIFE is target domain, and JOURNEY is source domain. Some structures of JOURNEY (beginning, ascent, descent, end, etc.) are considered in the given metaphor as a basis for the description of life structure. Similarly in other classical metaphor RICHARD - THE LION some 
lion qualities (for example, courage, but not tail, fangs, and claws) are transferred on a human being, who now becomes in frameworks of the metaphor domain.

An action of visualization metaphor consists of extractions of structures from target domain on the base of certain structures from source domain and transfer them in metaphor domain, which in this case has a visual nature. The visualization metaphor is mapping (more exactly operator) to certain visualization world, where unshaped objects get its visual presentations.

The use of metaphors should increase expressiveness of objects under investigation. To achieve it objects of target domain (with a set of structures, properties, and so on) are selected. As this takes place not all objects are chosen (and even not all their characteristic or structure elements), but only that, which are under interest most of all. Analogues for these objects (by way of structures, qualitative properties and so on) are searched in source domain. Further the following operation takes place. Object of target domain together with object from source domain are located in metaphorical domain, or more exact in doing so the metaphorical domain is generated. In this domain the investigated object now starts to function. (It is possible to consider, that it is already a new object of a new domain.) The metaphor domain gets autonomy from domains generated it. Many properties of its objects only mediately are connected (if at all are connected) to properties of source domain objects. There is a new logic of development metaphorical domain. So, for example, the use of the scientific metaphor of an electromagnetic field its intensity is studied. But it is obviously absent on a field of wheat. Thus, there is a projection of some target domain characteristics to source domain. And the task of metaphor completeness often may reduce quality of a metaphor.

There are questions - what are nature and structure of metaphor domain; how its generation is produced? The natural answer to them is connected to understanding of that the consideration of a metaphor as sign or as pair of signs is not fruitful. First of all the metaphor generates some sign system, that is integral sign set, in which existing internal relations between signs somehow map relations between designates. Our metaphor domain as a matter of fact is a sign system. In case of a metaphor the generation of a sign system is possible to consider as the adaptation of two metaphor operators, the basic: "Let A is similar to B" and the additional operator: "The following attributes /elements/characteristics of A are selected for assimilation to the following attributes /elements/characteristics of B" Where A is a source domain, and $\mathrm{B}$ is a target domain.

Other function of a metaphor is to define the context assisting to correct interpretation of elements of the given visualization language and to revealing of visual text meanings. Thus, a visualization metaphor is provided understanding of mapped entities of the application. Also a visualization metaphor participates in generation of new entities based on an internal logic of the metaphor.

\section{Metaphor Analysis}

Some different interface and visualization metaphors were analyzed. Among them popular desktop metaphor, room metaphor, and also metaphors used in highly specialized scientific and information visualization systems. We paid attention on 
genesis of metaphors, opportunities of data presentation and manipulation using concrete metaphors, potential properties of metaphorical objects, and also potential opportunities of user interpretation of these objects and manipulations with them. The purpose of analysis is to reveal structures of successful metaphors and to build a basis for comparison and evaluation of metaphors.

Let's define the concept "metaphor action". This characteristic is constructed by answers to the following questions:

"How it is possible to represent the information by this metaphor?"

"What do properties of metaphorical objects take place?"

"What does actions or ideas result from user interaction (including seeing) with metaphorical objects?"

Some sort of metaphor action "formula" is constructed on the base of analysis of the well-known interface metaphors.

It is possible to construct a "formula" of metaphor actions.

The metaphor "formula" may include simplified descriptions of source and target domains, an idea of likening using in the metaphor and results of metaphor actions.

In the case of desktop metaphor the formula may be written as follows:

Source domain: Desk with folders containing documents (documents are structured, but folders may be disordered);

Target domain: Office automation system;

Idea of likening: "Folders with papers" = "structure of the data, a set of files";

"Opening of a folder " = "demonstration of file structures and/or files";

"Processing of documents" = "execution of functions, by means commands of the visual language".

Result: The direct access to data structures by means manipulations of icons placed on the screen; calls of some [user] predetermined functions by means a visual dialog language.

Microsoft Windows uses the extended version of this metaphor.

Addition of source domain:

A desk is combined with control panel where starting buttons are placed.

Besides the "magic" idea is added: All actions within the framework of system are made by means of double click on icons.

Result: icons representing as data structures as programs calls.

There is also one more idea - opening of new windows when program executions are begun.

In other cases (for examples in case of room metaphor) we can't write the full metaphor formula because there are not common application domain, successful and generally used experience of its usage, and, that is there is not unities of target domain. However we shall result the review of properties of room metaphor. Realizations of this metaphor are characterized often by a combination of threedimensional space of the room with bidimensionality of objects, placed on its "walls", a "ceiling" or a "floor". Such combination on the one hand preserves principles of structural correspondence between model entities and visual objects, on the other - provides successful spatial placement of images.

Let's carry out the analysis of a room metaphor from two positions: the room metaphor itself and how it is possible to represent the information with its help.

The room metaphor possesses the following properties: 
1. Ability to contain any objects inside itself.

The room not only represents separate object, but also is the container for others ones.

2. Restriction of a perception context. Objects inside a room are considered in a separation from "external worlds".

3. Closeness. There are no any additional elements to use Room metaphor (excepting possible inner objects).

4. Inclusion in structure. It is possible "to build buildings of rooms", that is to consider set of rooms. Therefore the room may be an element of construction of some complex construction.

5. Naturalness of a metaphor. The room is natural metaphor, with presence of corresponding objects in the real world. Functionality and characteristics of real objects are transferred in the virtual world with only minor extended understanding.

The room metaphor was used to extend the possibility of desktop metaphor in office automation systems. But more effective in this case is using the combination of the main (desktop) metaphor and local metaphor reflecting as the specialization as user's mentality and labor experience. We use the idea of card file as local metaphor for municipal clerks. In this card file metaphor also some "magic" (automatic) operations are used to improve searching and ordering of documents. Thus, it is possible to characterize the complex metaphor "work with a card file placed on the desktop". This metaphor is natural for users. Also it is important the presence of structure in frameworks of the metaphor. The concept of a card file means structuring of the data. Use of this metaphor supports user mental models and has positive psychological effect. The user may be sure that she/he will find quickly the necessary information in structured repository. This metaphor (as well as real object) has organizing information functions. The card file metaphor allows using the concrete office automation system more effectively. This local metaphor structures as visual representation of programs, and user's operational activity. Explicit usage of the metaphor helps systematic designing of the interface.

In the majority of the specialized visualization systems the representations of abstract per se mathematical, program, information objects takes place. For a part of problems habitual views are used. However there are cases, when existing visualization methods are not applicable. Then it is necessary to search metaphorical source domain. Frequently this search is rather serious problem. Revealing of all components of metaphors in these cases is a difficult task. However there is our experience of search of new metaphors for scientific, information and software visualization systems [3]. For example the complex visualization metaphor was suggested to represent four-dimensional set resulted modeling of complex chemical processes [18].

\section{Metaphor Structures and Analysis of Metaphor Generation}

The analysis of results of metaphors and views transitions in different visualization domains has shown the presence of some regularity independent of visualized information nature. This regularity determines a success or a failure of visualization 
techniques. To achieve a success it is necessary the connection between the internal nature and structure of modelling objects (and the data corresponding to them) and internal mental structures of users. In this structure (so called "representative cognitive structures") his/her image of phenomena is mapped. Correspondents between model and cognitive structures are not arbitrary.

The person distinguishes any general logic in a picture, breaking it on set (perhaps enclosed) fragments, abstracting from minor elements. Thus, it is possible to speak about cognitive structures, structures of entities under analysis and structures of visual objects and images. When analysing a visual image interpretation proceeds in two phases. The elements interpreted according to the knowledge extracted from a dedicated domain are revealed. The information on interrelations between these elements is extracted (reconstructed) from the general visual image. Thus, a coherent adequate representation about visualized object is provided. That is the interpretation proceeds in part on the base of the visual image itself, and in part on the base of the analysis of its elements and their interpositions.

The information on element interrelations specifies the structure of a visualized object or more exact, our presentation about it. Process of visualization may be considered as construction of visual (geometrical) image on the basis of abstract representation about object. These abstract representations are model (object, the phenomenon, process under investigation), somehow connected with user cognitive structures describing a given entity. Visual images representing modelled entity, serve to create or extract by it cognitive structures. The purpose of researches in visualization domain is to create such techniques and principles, which will provide the extraction adequate cognitive structures on visual images. A process of interpretation is exactly the generation of cognitive structures on base of visual images. This process is inverse or more exactly dual to visualizations. As visualization principles the interpretation principles should exist.

Revealing of modelling (initial) object structure may reduce the complexity of development of a view representing this object. In this case the complex view may be developed as compound view. Therefore a criterion of views correctness is necessary. In this case construction of views may be based on such rule, that structure of the constructed visual image should not contradict to the structure of the initial entity. During the interpretation of visual objects there should not be relations absent in the initial entity. For example representation of the modelling entity with a bidimentional structure as 3D object may lead to wrong interpretation. Though often the increase of an image dimension are useful for better interposition of represented objects in a scene. While a reduction of dimension, as well as a reduction of all structures is not a mistake provided that the user (interpreter) is informed about it.

There is the question, why one metaphors (not only computer, but also literary or rhetorical) appear successful, despite of obvious discrepancy, and others, exact and detailed, are unsatisfactory? The answer we shall search considering the process of metaphor generation.

The goal here is to define relation between metaphor and target system of meanings, for which metaphor was formed. This relation might be expressed in the terms of "meanmarks". To define what meanmark is, let us consider the word "brave". The meaning of that word may be established through if-then (implication) relation of it to another words. The implication is important because it is base for 
reasoning, and metaphors help the process. For the word "brave" one may define outgoing implications. For instance, if A is "brave" then A is "not timid". This relation is denoted with ->, "brave" -> "not timid". Besides outgoing arrows every word has incoming ones.

So the following object emerges. I -> "brave" $->0$, where I is set of words implying "brave" and 0 is set of those which are implied by "brave" itself. Now the graph of such implications can be considered, and it is assumed here that the topology of graph's paths passing through node defines mean of that node. Meanmark is simple the label for node in such oriented graph. The graph may not be the graph of implication relations of words, it may depict any such relation.

Both metaphorical system of meanings and target system have such graphs which show how meanings are connected within them. According to that representation the correspondence between meanings at target system and meanings at metaphor can be established through homomorphism from target system meanmarks graph onto meanmarks graph of metaphor.

Let us consider example. If we want to illustrate the target system "King Richard is brave", firstly we must define meanmarks graph of that system. It is simple enough: Richard -> king, brave. But the meanmarks "king" and "brave" mean nothing without their language contexts: incoming and and outgoing implications in English's system of meanings. It is very difficult to find meanmarks in some graphs which will have the same topology of implications as "king": and "brave" in that system. So required metaphor should include both these meanmarks. We are looking now for "somebody" -> king, brave. And, of course "somebody" is "Lion:".

That is meanmarks graph is constructed. This graph may be superimposed on the similar graph, consisted of concepts of source domain. It is natural, that a set of meanmarks in this graph is distinct from source one, however its general structure is similar. Searching of a metaphor - is searching of the structure of interrelations which are similar to structure of interrelations in the target domain. Superimposing (not exact, not one to one) may be, that some of the bottom levels will be necessary to remove. But the additional level (absent in a target domain) may be appear. This second system of meanmarks with its interrelations is metaphor of an image considered originally. Interrelations between concepts in metaphorical system may be richer than in target one. Some interrelations may correspond to those arrows, which are present in the first set, but have not been noticed up in stage of metaphor construction. Here they may be obviously appreciable.

The suggested model may also help to describe how metaphor allows to establish new properties of target system.

Let's assume, that we have certain time sequence of values $\{X\}$. That is $\{X\}$ is a set of elements with one linear discrete coordinate and some value. Problem of presentation of this sequence is raised. For example, let's transform these values into music notes with certain melody. It is possible because notes may be interpreted as values, and duration of music notes is always discrete. Then:

Melody -> time sequence of notes $->$ a set of elements with one linear discrete coordinate and a certain value.

Recognition $\{\mathrm{X}\}$ through a similar metaphor may lead to interesting conclusions. The sequence of notes has the special property - notes may compose (or not) to beautiful melodies. And if our way of formal transformation values $\{X\}$ to notes may 
generate melody, that, probably, $\{\mathrm{X}\}$ has interesting properties, which may be found out by means of a metaphor.

The process of metaphor generation (metaphorization) first of all includes (may be implicit) analysis of target domain of the future metaphor. On a basis of the metaphor objects and its properties the hierarchical structure of object interrelations of target domain and their properties reveals. At the following stage a source domain and its main object are searched. Criteria of a choice are criteria of metaphor quality.

The success of a metaphor is connected first of all with structure of interrelations concepts in source domain and with an opportunity to understand relations in the target domain. The structural analysis of metaphors helps to construct criteria of metaphor quality.

\section{Conclusion}

Let's consider a set of requirements to a choice of metaphors and views.

The metaphor has to generate integral and systematic interface and/or visualization systems. It results an undesirability of the interface based on several fine metaphors describing details and components of dialog. This requirement means also necessity to conform as to others already existing computer metaphors, and to general ideas global metaphors.

Reduction of interpretation complexity is considered as a condition of "good" metaphor. Therefore direct interpretation of images is required, but decoding and interpretation of complex [visual] texts is considered as a source of failures.

Set of the criteria, imposed on initial and target domains in a process of metaphor generation includes:

- similarity of object properties in source and target domains,

- ability to visual presentation for object in the source domain,

- habitualness (recognition) of objects in the source domain,

- rich set of interrelations between objects in the source domain.

Also we may write criteria of generation for views based on metaphors. Among them such, as truthfulness, laconicalness, expressiveness, clearness.

Researching metaphors, views and their structures allows to analyze and evaluate existing design decisions and to project interactive visual systems with necessary properties.

\section{References}

1. Averbukh V.L. Visualization Metaphors. Programming and Computer Software. Vol. 27, No. 5, 2001 pp. 227-237.

2. Averbukh V.L. Towards the Conceptions of Visualization Language and Visualization Metaphor. Proceedings of IEEE Symposia on Human Centric Computing Languages and Environments: September 5-7, 2001 Stresa, Italy. IEEE. 2001. P. 390-391.

3. Averbukh V.L. Toward Theory of Computer Visualization Computational Technologies V.10, N 4, 2005, pp. 21-51. (In Russian.)

4. Averbukh V.L. HCI Metaphor and Visualization Metaphor. What Theory Does We Need? The 16th International Conference on Computer Graphics and Applications 
GraphicCon'2006, July 1-5, 2006 Novosibirsk, Akademgorodok, Russia Conference Proceedings. 262-268. (In Russian.)

5. Bajdalin A., Ismagilov D. Structure Representation in Software Visualization Systems. The 16th International Conference on Computer Graphics and Applications GraphicCon'2006, July 1-5, 2006 Novosibirsk, Akademgorodok, Russia Conference Proceedings. 271-274 (in Russian.)

6. Barbosa S.D.J., de Souza C.S. Extending software through metaphors and metonymies. Proceedings of the 5th international conference on Intelligent user interfaces. January 09-12, 2000, New Orleans, Louisiana, United States. P. 13-20,

7. Barr P. Noble J., Biddle R. A Semiotic Model of User-Interface Metaphor. Chapter in Virtual Distributed and Flexible Organisations. Edited proceedings of the Sixth International Workshop on Organisations Semiotics in Reading, UK. Kluwer Academic Publishers, 2003. http://www.mcs.vuw.ac.nz/ chikken/research/papers/iwos2003/barr \iwos2003.pdf

8. Blackwell A. The Reification of Metaphor as a Design Tool. ACM Transactions on Computer-Human Interaction (TOCHI) Volume 13, Issue 4 (December 2006). 490-530.

9. Brown Th. L. Making Truth: Metaphor in Science. Urbana Champaign. University of Illinois Press, 2003.

10. Gazendam H.W.M. Information System Metaphors (Groningen University, NL \& Twente University, NL)

http://www.econ.uba.ar/servicios/publicaciones/journal3/ ontents/HGazendam/methaphors.htm

11. Kuhn T. Metaphor in science. In: Metaphor and thought. Ortony A. ed. Cambridge: Cambridge University Press. 1979. Pp. 533-542.

12. Kuhn W., Frank A.U. A Formalization of Metaphors and Image-Schemas in User Interfaces. Cognitive and Linguistic Aspects of Geographic Space, NATO ASI Series, Kluwer Academic Publishers, 1991. Pp. 419-434

13. Lakoff, G. (1993). The contemporary theory of metaphor. In A. Ortony (Ed.), Metaphor and Thought (2nd ed., pp. 202-251). Cambridge: Cambridge University Press.

14. Lakoff G., Johnson M. (1980). Metaphors we live by. Chicago: University of Chicago Press.

15. Musil S. Monitoring Parallel Programs with INHOUSE http://www.ani.univie.ac.at/ani/research/Monit.html

16. Richards S., Barker P., Banerji A., Lamont C., Manji K. The Use of metaphors in iconic interface design. Intelligent Tutoring Media 1994. Volume 5. Number 2, pp. 73-80.

17. Simoff S.J. Towards the development of environments for designing visualisation support for visual data mining. Proceedings International Workshop on Visual Data Mining, 2001, pp. 93-106.

18. Vasev P.A., Pervalov D.S. One method of visualization of four-dimension sets. Proceedings of conference "Supercomputing technologies in physical and chemical researches", Chernogolovka, Russia, 2001, pp. 32-36. (in Russian.) 